\title{
High Throughput Quantitative Phase Mapping Using Synchrotron X-Ray Diffraction
}

\author{
Jae-Hyuk Her ${ }^{1}$, Yan Gao ${ }^{1}$, Erik Jezek ${ }^{1}$, Job Rijssenbeek ${ }^{1}$, Hui Zhong ${ }^{2}$ and John Parise ${ }^{2}$ \\ 1. GE Global Research Center, Niskayuna, NY 12309, U.S.A. \\ 2. Department of Geosciences, Stony Brook University, Stony Brook, NY 11794, U.S.A.
}

The X-Ray diffraction (XRD) technique has been used to characterize crystalline phases in mixtures ever since its discovery. In contrast to the spectroscopy, it can provide quantitative phase information via Rietveld refinement [1]. While spectroscopy is commonly used to map surface elemental distributions, XRD has not been popular for such mapping applications - likely due to the lack of suitable instruments and analysis software. Nowadays, high brilliance and high energy synchrotron Xrays are available, that can penetrate relatively thick bulk materials. And with highly collimated micronsize beams, the technique can generate tens of thousands diffraction patterns in a few hours - which can cover a few $\mathrm{cm}^{2}$ surface with sub-mm resolution. Quantitative analysis of such big data sets remains a challenge since most Rietveld refinement programs were not developed with such applications in mind.

Motivated by a desire to better understand the aging mechanisms in GE's Durathon ${ }^{\mathrm{TM}}$ sodium metal halide batteries (NaMx), we developed a high throughput phase quantification method to map the phase distribution in these thick cathodes at different stages of charge and discharge. High throughput was implemented in the TOPAS program [2] using its batch Rietveld refinement programming capability. All results of batch processing were saved into a master text file, from which phase fraction values were extracted afterwards. Finally, phase fraction values were converted to a format compatible with graphics programs (e.g., Microsoft Excel), and phase maps were reconstructed in the shape of original cell for better comparison.

Each cathode sample was about $6 \mathrm{~mm}$ thick, and multiple samples were packed into a single package for the XRD experiments. The synchrotron data were collected at Beamline X17A, National Synchrotron Light Source (NSLS), Brookhaven National Laboratory, and Beamline 11-ID-B, Advanced Photon Source (APS), Argonne National Laboratory. The measurements were performed in transmission mode, where $68 \mathrm{keV}$ (NSLS) and $58 \mathrm{keV}$ (APS) X-rays, $0.5 \times 0.5 \mathrm{~mm}$ in cross-section, penetrated through the bulk samples. The diffraction data, averaged along the beam path (sample thickness), were collected by a two-dimensional detector at both synchrotrons. The data collection was automated to raster over an area of about $40 \times 50 \mathrm{~mm}$ in $0.5 \times 0.5 \mathrm{~mm}$ steps, generating more than 8000 diffraction patterns in 8 (NSLS) to 2 (APS) hours. The 2D data were reduced into analyzable raw 1D XRD patterns using the program Fit2D [3].

Our method successfully visualized quantitative phase maps of the cathodes from tens of thousands of raw XRD data sets (Fig. 1). Analysis of the resulting phase maps identified areas of high and low electrochemical activity (via the presence of $\mathrm{NiCl}_{2}$ ) and thus informed our hypotheses of degradation. Particularly, the non-uniform distribution of $\mathrm{NiCl}_{2}$ in the cathode (Fig. 2a) suggested that the active materials were transported over long distances and were not effectively utilized. The overly chlorinated outer regions in early cycles (Fig. 2a, left) presaged later degradation that eventually left only a small amount of active materials near the center of the cell (Fig. 2a, right). More recent designs have mitigated such non-uniform utilization (Fig. 2b), significantly reduced the capacity fade and improved overall 
battery life.

Beyond batteries, this work demonstrated how synchrotron radiation can help industry solve key technological problems, and highlighted the power of big data analysis for advanced materials characterization [4][5].

[1] Rietveld, H.M., J. Appl. Cryst., 22, 65-71, 1969

[2] Bruker Corporation

[3] Hammersley, A.P., ESRF Internal Report, ESRF97HA02T, 1997

[4] The authors gratefully acknowledge funding support from the New York State Energy Research and Development Authority (NYSERDA) via the New York Battery and Energy Storage Technology Consortium (NY-BEST). NYSERDA has not reviewed the information contained herein, and the opinions expressed in this report do not necessarily reflect those of NYSERDA or the State of New York.

[5] The authors deeply appreciate Dr. K. Chapman's help in the experiments done in Beamline 11-ID-B, Advanced Photon Source, Argonne National Laboratory. This research used resources of the Advanced Photon Source, a U.S. Department of Energy (DOE) Office of Science User Facility operated for the DOE Office of Science by Argonne National Laboratory under Contract No. DE-AC02-06CH11357. Use of the National Synchrotron Light Source, Brookhaven National Laboratory, was supported by the U.S. Department of Energy, Office of Science, Office of Basic Energy Sciences, under Contract No. DE-AC02-98CH10886.
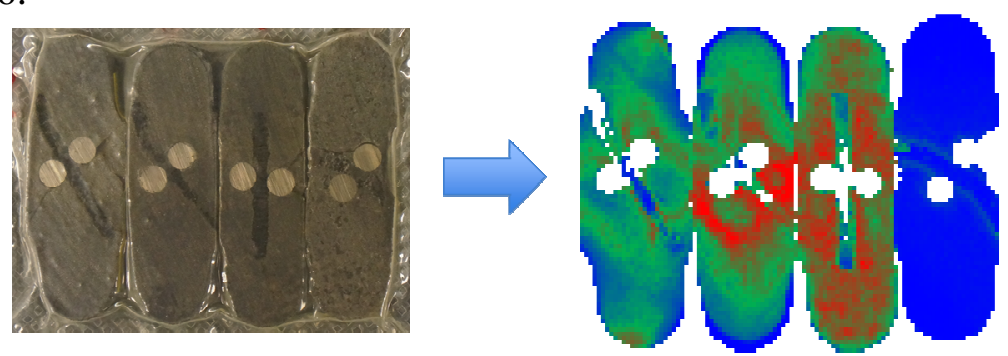

Figure 1. Optical image of sliced samples from 4 different $\mathrm{NaMx}$ cathodes (left) and their corresponding quantitative $\mathrm{NiCl}_{2}$ abundance maps (right). The actual dimension of the set of 4 samples is approximately $40 \mathrm{~mm}(\mathrm{H}) \times 50 \mathrm{~mm}(\mathrm{~W}) \times 6 \mathrm{~mm}(\mathrm{D})$.

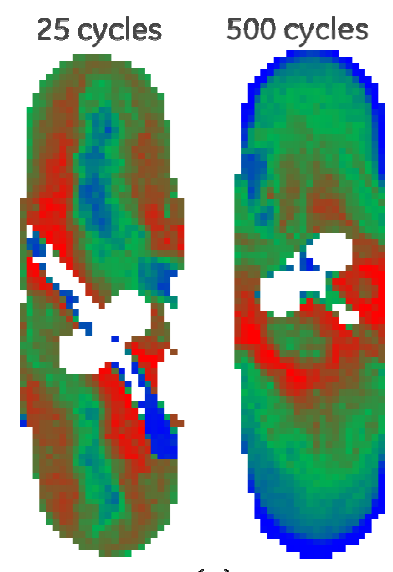

(a)

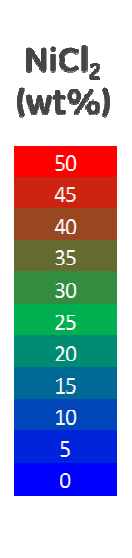

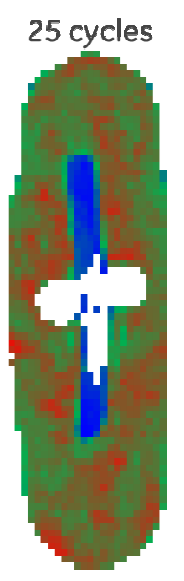

(b)

Figure 2. Quantitative $\mathrm{NiCl}_{2}$ phase maps of cathode slices from cells with (a) a higher degradation design and (b) a lower degradation design after 25 and 500 accelerated test cycles. Colors correspond to the weight percent values shown in the scale bar in the middle of the figure. 